Aim of the study: Radical nephrectomy in the treatment of renal cell carcinoma (RCC) remains the gold standard, but nephron-sparing surgery (NSS) is still increasing in importance. The main goal of this study was to compare the diagnostic accuracy of ultrasound and multi-detector computed tomography in RCC staging and its influence on deciding about further patient treatment. Material and methods: 87 patients (age range 27-90 years; median 61.5) underwent ultrasound (US) scan and contrastenhanced computed tomography (CE-CT) of the abdomen and pelvis. 28 patients were qualified for NSS. The remaining group of patients underwent nephrectomy.

Results: NSS was performed more frequently among patients with lesions in the lower pole of the kidney and there was no infiltration to the calyx and renal pelvis. Radical nephrectomy (RN) was pursued in cases with lesions in the central or upper pole. Lesion diameter in patients qualified for NSS was smaller than in patients qualified for radical nephrectomy.

Conclusions: Determining the relationship between tumour and adjacent structures is not a simple matter. According to our study, $50 \%$ of $\mathrm{CT}$ results differ from histopathology assessment. Tumour diameter determined in CT examination is larger than in ultrasound and histopathological measurements while US scanning tends to underestimate tumour size in relation to histopathological assessment.

Key words: renal cell carcinoma, nephrectomy, nephron sparing surgery, US, CT.

\section{Nephrectomy or nephron-sparing surgery - how to decide?}

\author{
Elżbieta Łuczyńska ${ }^{1}$, Sonia Dyczek ${ }^{1}$, Sylwia Heinze-Paluchowska ${ }^{1}$, \\ Artur Komorowski', Tomasz Pawlik ${ }^{1}$, Wojciech Wysocki ${ }^{2}$, Małgorzata Klimek ${ }^{3}$
}

1Departament of Diagnostic Imaging, Maria Skłodowska-Curie Institute of Oncology, Cracow; Department of MRI, IFJ PAN, Cracow, Poland

2Department of Surgical Oncology, Maria Skłodowska-Curie Memorial Cancer Center and Institute of Oncology, Cracow Branch, Poland

${ }^{3}$ Laboratory of Oncological Genetics, Maria Skłodowska-Curie Memorial Cancer Center and Institute of Oncology, Cracow Branch, Poland

\section{Introduction}

Among genitourinary tumours, renal cell carcinoma (RCC) is the third in frequency and the first in morbidity among urological cancers; $3 \%$ of all malignant tumours in adults are renal cell carcinomas [1]. Tobacco smoking, exposure to certain chemicals and genetic factors in some cases (von Hippel-Lindau syndrome) are the most important risk factors [2].

Stage, size and localization of the tumour are the most significant prognostic factors influencing survival rate, as the only effective therapy for renal cell carcinoma is an adequate surgical procedure [3, 4]. Accuracy of cancer staging is of the greatest importance for preoperative planning and further prognosis. Open nephron-sparing surgery requires knowledge of renal and tumour vascular supply as well as of the tumour's relationship to the collecting system and adjacent normal renal parenchyma, which are essential in mapping out an operative strategy $[5,6]$. Determining the range of inferior vena cava invasion is crucial, as it might warrant more extensive surgery in patients with intraluminal tumour thrombus [7, 8].

The widespread availability of abdominal ultrasound and computed tomography (CT) scanning has increased the diagnosis of incidental renal tumours, which now comprise the vast majority of new cases diagnosed each year [9]. Due to better availability of two imaging methods, ultrasonography (US) and conventional tomography (CT), in the last two decades, detection of RCC has significantly increased. The average size of detected lesions was smaller and their stage was lower, compared to previous average results. What is more, detection of asymptomatic lesions is also possible due to application of those two diagnostic modalities, which further results in a better survival rate [10, 11].

\section{Aim of the study}

The aim of this study was to compare the diagnostic accuracy of ultrasound (US) and multi-detector computed tomography (MDCT) in renal cell carcinoma staging. In 1969 a therapeutic standard for renal cell carcinoma was established by Robson, who performed radical nephrectomy including ipsilateral adrenalectomy [3]. With the development of new surgical techniques another procedure, open nephron-sparing surgery (NSS), appeared to bring favourable results [12-14]. At first that procedure was suitable for patients with a lesion in a solitary kidney or with bilateral tumours. Nowadays, it is approved in elective cases and considered as a safe and effective surgical procedure, which is accessible according to recent development of new imaging techniques, such as MDCT followed by advanced image reconstruction. It allows for accurate estimation of the ratio of tumour dimensions to calyx, renal pelvis and surrounding tissue $[15,16]$. 


\section{Material and methods}

Eighty-seven surgical procedures among patients with renal tumour (45 men and 42 women) were performed in 2007 and 2008. The patients' age ranged from 27 to 90 years (median 61.5). All of the patients underwent staging diagnostic examinations including ultrasonography scan US (Fig. 1) and contrast-enhanced computed tomography (CECT) of the
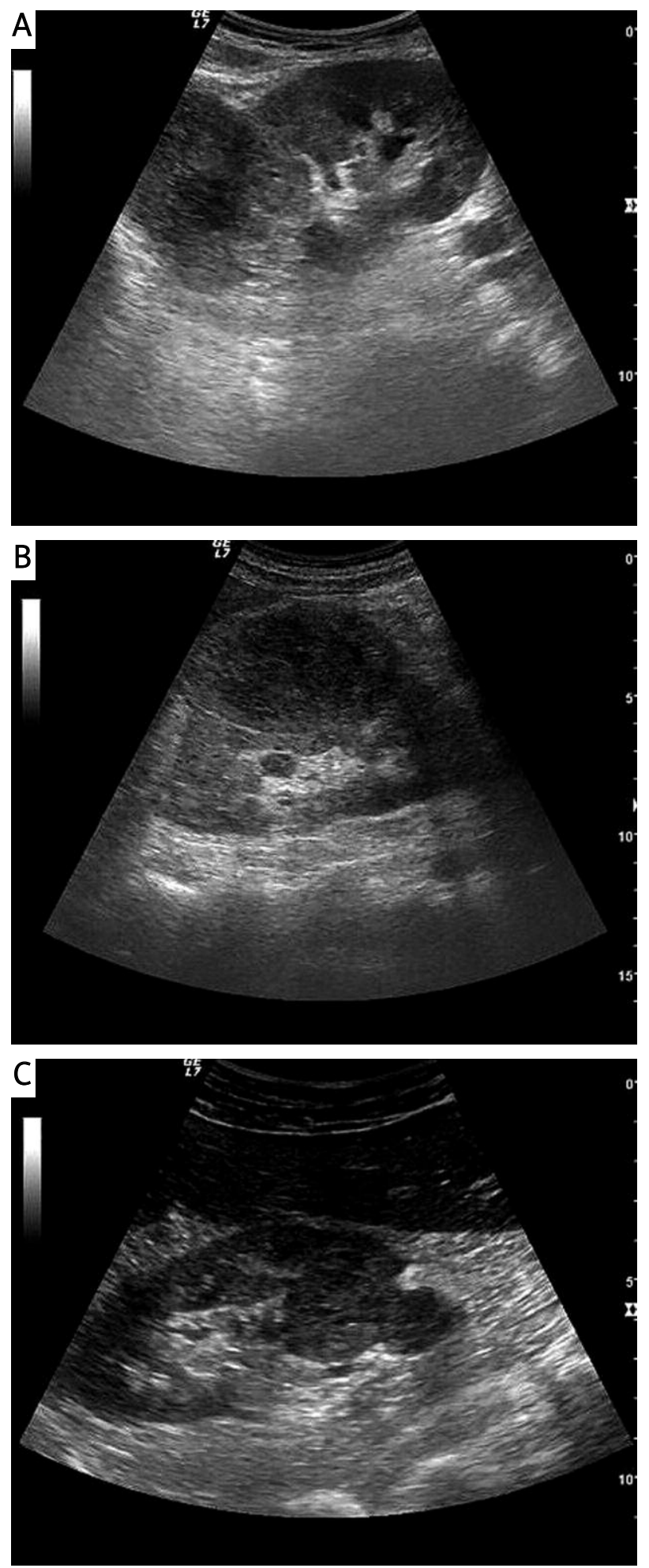

Fig. 1. US scans of the abdomen (lesion localised $\mathbf{A}$ - in the lower pole; $\mathbf{B}$ - in the central part; $\mathbf{C}$ - in the upper pole of the kidney) abdomen and pelvis (Fig. 2). The trans-abdominal gray-scale US scan was performed using the GE $4 \mathrm{MHz}$ Convex Transducer with Doppler application. Computed Tomography was performed using a GE LightSpeed 16-Slice Multidetector Scanner (290 mAs, 120 kV, 5.0 mm slice thickness, Ultravist intravenous contrast agent - dose $60 \mathrm{ml}$ administered at a rate of $3 \mathrm{ml} / \mathrm{s}$ ). After completion of $\mathrm{CT}$ examination, the MPR reconstructions of the axial images were obtained in the sagittal and coronal planes (Fig. 3).

In both examinations the following features were estimated: tumour diameter $(\mathrm{mm})$, position of the tumour in relation to the renal pelvis and calices, infiltration of adjacent tis-
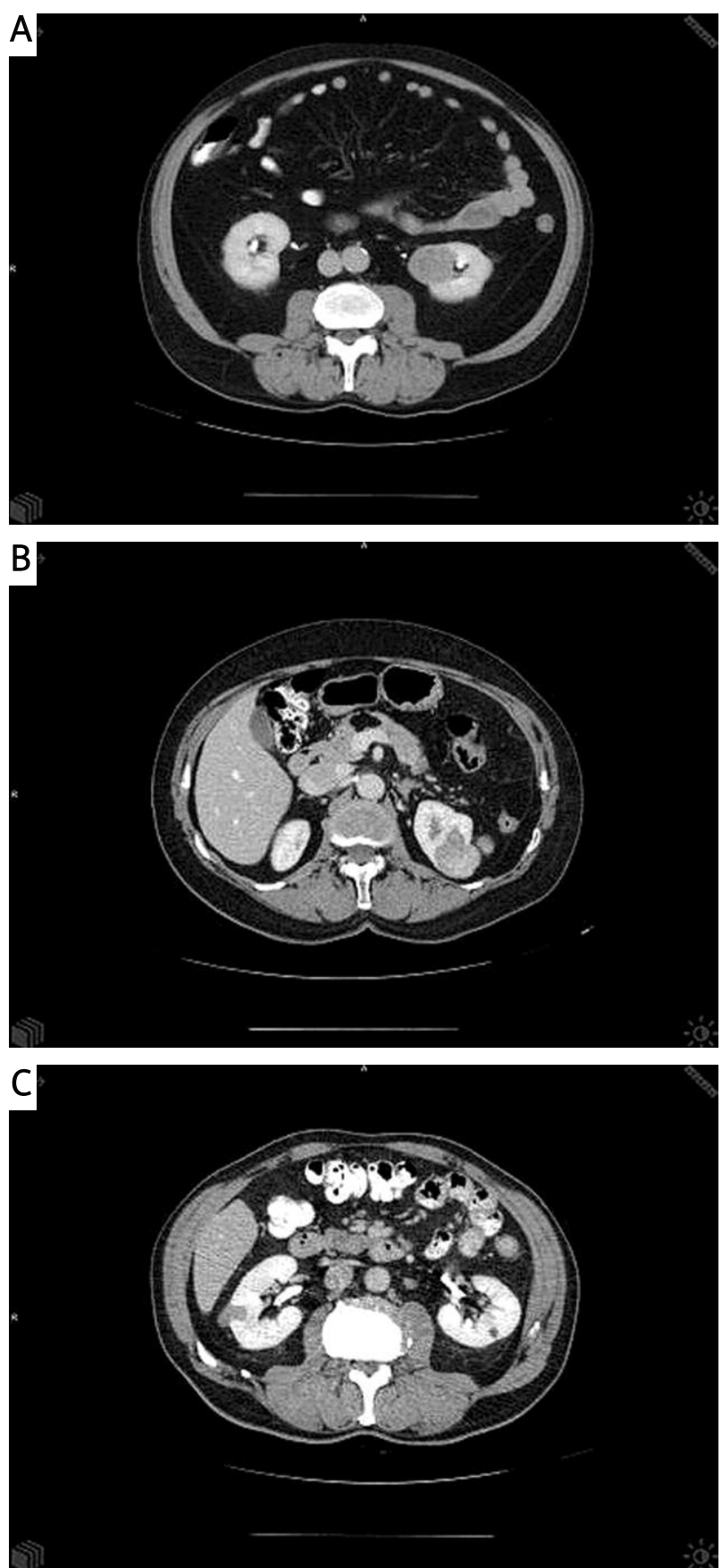

Fig. 2. CT axial images of the abdomen (lesion localised $\mathrm{A}$ - in the lower pole; $\mathbf{B}$ - in the central part; $\mathbf{C}$ - in the upper pole of the kidney) 

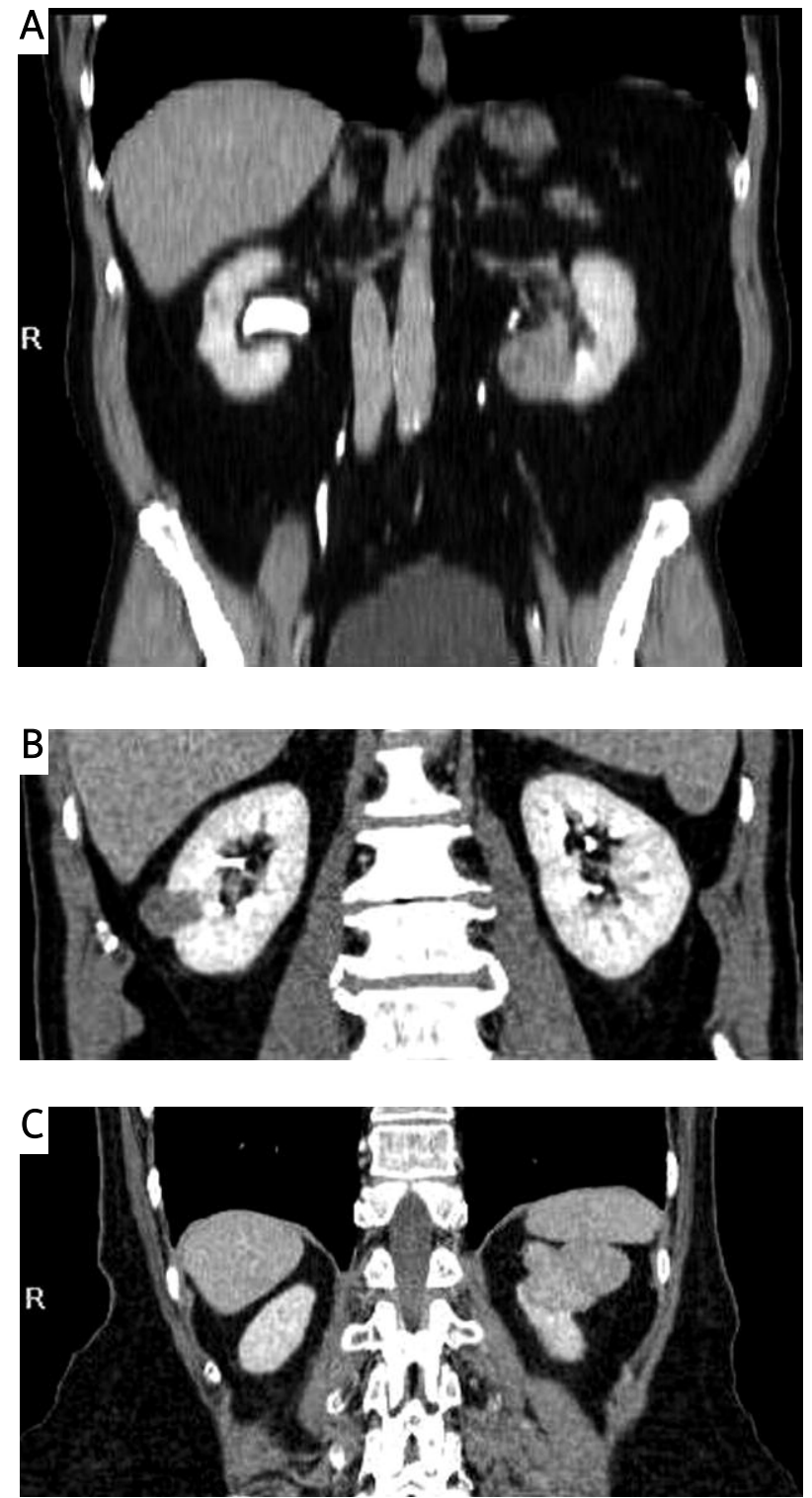

Fig. 3. Coronal reconstructions of CT images of the abdomen (lesion localised A - in the lower pole; B - in the central part tumour diameter vs. type of the imaging modality

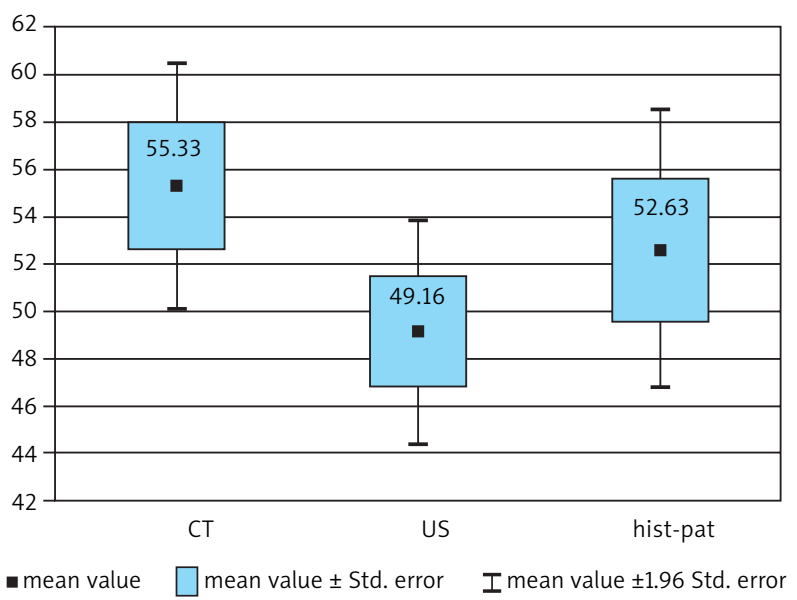

Fig. 4. Relation between tumour diameter and type of imaging modality

sues, presence of thrombus in renal vein and inferior vena cava, and tumour's Hounsfield absorption factor before and after contrast administration. Statistical analysis was performed with STATISTICA vs. 9. In all statistical procedures, $p<0.05$ was considered significant.

\section{Results}

Analysis of our material showed the following results. Size of the tumour estimated in ultrasound ranged from $10 \mathrm{~mm}$ to $106 \mathrm{~mm}$ (average $49 \mathrm{~mm}$ ) and in CT from $10 \mathrm{~mm}$ to $120 \mathrm{~mm}$ (average $55 \mathrm{~mm}$ ).

Tumour diameter determined in CT examination was greater than in ultrasound and histopathological measurements (Fig. 4).

CT scan tends to underestimate tumour size in relation to histopathological assessment in patients qualified for radical nephrectomy and nephron-sparing surgery alike (Fig. 5).

Analyzing the data we found that NSS was performed more frequently among patients who had lesions localized in the lower pole of the kidney $(p=0.014)$, and radical nephrectomy was
A

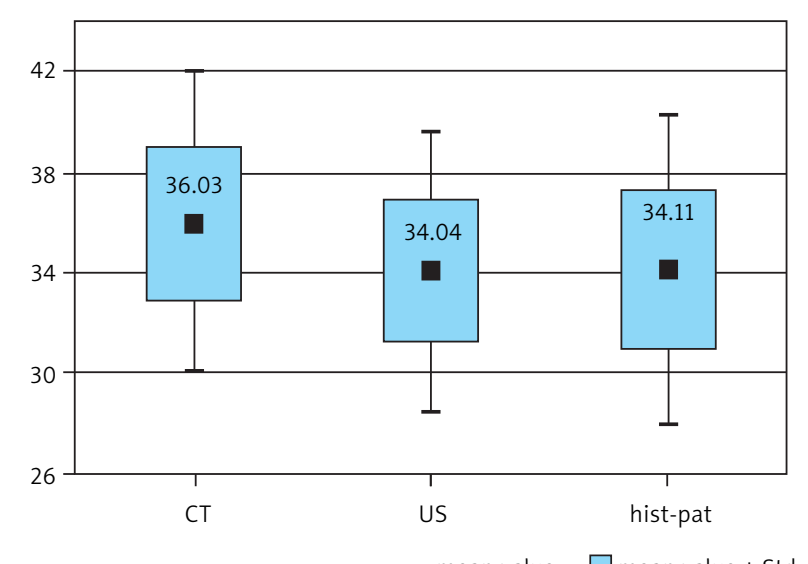

B

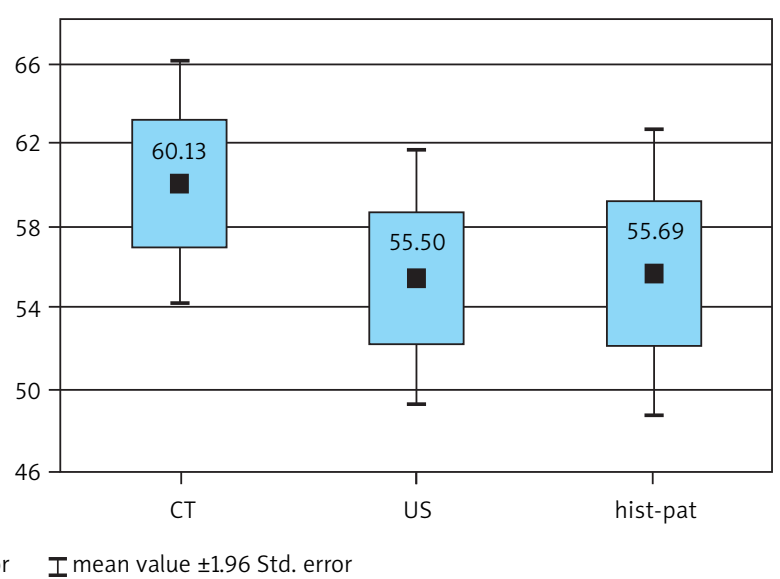

Fig. 5. Tumour diameter vs type of imaging modality. A - nephron-sparing surgery (NSS); B - radical nephrectomy (RN) 
Table 1. Proportional distribution of lesion localization related to imaging modality and type of surgical procedure

\begin{tabular}{lcccc} 
Imaging modality & \multicolumn{4}{c}{ Lesion localization in NSS } \\
\cline { 2 - 4 } & Upper pole & Central kidney & Lower pole & Peripelvic location \\
CT & $14.29 \%$ & $32.14 \%$ & $53.57 \%$ & $0 \%$ \\
US & $14.29 \%$ & $32.14 \%$ & $53.57 \%$ & $0 \%$ \\
& & Lesion localization in radical nephrectomy & \\
\cline { 2 - 4 } & Upper pole & Central kidney & Lower pole & Peripelvic location \\
CT & $35.59 \%$ & $33.90 \%$ & $22.03 \%$ & $8.47 \%$ \\
US & $29.31 \%$ & $36.21 \%$ & $25.86 \%$ & $8.62 \%$
\end{tabular}

performed in cases of lesions localized in the central part $(p=0.11)$ or upper pole $(p=0.08)$ of the kidney (Table 1$)$.

Infiltration of the renal collecting system was found in 33 patients in computed tomography examination. Among patients qualified for nephron-sparing surgery due to CT examination results infiltration of the calyx and renal pelvis was not detected in the majority of cases $(p=0.00)$, opposite to radical procedures, where infiltration of the calyces is much more common $(p=0.02)$ as well as renal pelvis infiltration $(p=0.05)$ (although in the case of renal pelvis infiltration it was of borderline significance) (Fig. 6).

In CT many factors, including tumour diameter, lesion localization and relation to calyces, were taken into consideration. In NSS tumour diameter was smaller than in RN (mean value in NSS was $36 \mathrm{~mm}$ while in RN it was $60 \mathrm{~mm}$ ). CT examination revealed adjacent tissue infiltration among 10 patients (with 2 cases of liver involvement and all other cases of adipose tissue infiltration). However, ultrasound scan confirmed adjacent tissue infiltration among 4 patients ( 2 cases of liver infiltration and 2 cases of adipose tissue infiltration). The pathology report revealed fibrous capsule infiltration in 17 patients, adipose tissue penetration in 13 patients and liver involvement in 1 patient, of those who had undergone radical nephrectomy.

When comparing these two imaging modalities (CT and US), their ability to detect vessel thrombus presence should be taken into consideration.

\section{Discussion}

Renal cell carcinoma (RCC), a potentially curable lesion, is the most common primary renal malignancy. Due to the importance of early diagnosis and the lack of specific clinical features of RCC, imaging plays a vital role in the diagnosis and management of RCC [17].

Ultrasound is the first, basic examination which allows for detection of abnormalities within the kidneys. US usually is the first imaging technique used to evaluate patients with suspected RCC. Its accuracy relies on the skill and experience of the ultrasonographer and on the patient's constitution [18]. Lesions which are found accidentally during ultrasound examination and are diagnosed as renal cell carcinomas are often smaller, better differentiated, of a lower stage, and have a better prognosis than those diagnosed on the basis of classic triad symptoms (haematuria, pain, palpable mass) [19].

Acquired data were analyzed and it was found that in ultrasound examination we can estimate the size of the lesion, its echogenicity, and in more advanced cases infiltration of adjacent structures. However, it is difficult to describe the

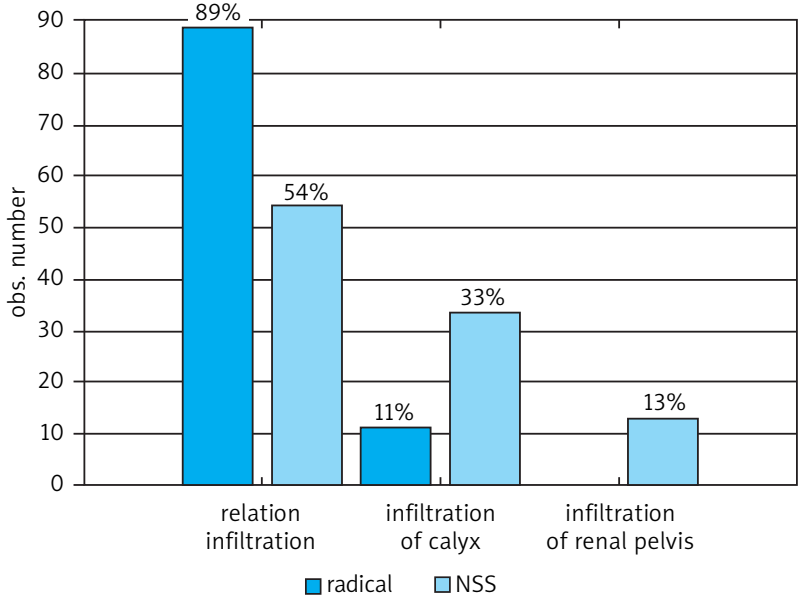

Fig. 6. Relation to calico-pyelo system

relation of the lesion to calices or the renal pelvis or infiltration of these structures. Despite the constraints of US in providing a complete evaluation of renal tumours before treatment planning, initial screening, characterization of renal masses and staging of RCCs can benefit from some recent advances in the technique.

One of the most relevant clinical advantages of US is the increased early detection of RCCs. Recent technical improvement of grey-scale imaging has enhanced US performance in the detection of small renal tumours. Ultrasound contributes additional diagnostic information for differential diagnosis of some renal masses that remain equivocal in CT, including atypical cystic lesions and angiomyolipomas with a minimal fat component. Ultrasound may also provide additional diagnostic information over CT in selected cases of RCCS with venous invasion [20].

However, the previously mentioned limitations of US were the main reasons why $\mathrm{CT}$ has become another basic method used for renal lesion characterization.

Contrast-enhanced CT is a standard imaging modality for renal tumour staging and for planning the site and area of resection. It has higher accuracy than ultrasound, especially in assessment of renal vein lesions and adjacent tissue infiltration [21]. CT remains the most appropriate imaging modality to differentiate benign from malignant lesions. Although RCC can appear as iso-, hyper- or hypodense lesions on native CT scans, it usually demonstrates a significant contrast enhancement of about $115 \mathrm{HU}$ and intratumoural areas of necrosis following the intravenous application of contrast medium [22]. 
Newer imaging modalities have superseded plain-film radiography in the diagnosis of RCC by offering greater sensitivity and specificity in both diagnosis and staging [23]. RCC can be diagnosed with CT with greater than 95\% accuracy. Once an RCC is detected, tumour staging becomes paramount for treatment planning [21, 24, 25].

In 2001 the first publication about the application of CECT in planning NSS for renal cell carcinoma appeared and the advantages of gap-free reconstructions in volume rendering and other 3-dimensional post-processing methods were discussed. This study was focused on the application of multidetector CECT and ultrasound in the determination of a tumour's local extension and infiltration of renal structures such as veins, arteries and the collecting system.

When deciding whether treatment should be NSS or radical nephrectomy, one of the deciding factors is the size of the tumour. Until recently the borderline was set at $4 \mathrm{~cm}$, but nowadays it is challenged by resecting tumours up to $7 \mathrm{~cm}$. Based on our material we conclude that CT tends to overestimate size of tumours compared to histopathology and from this we conclude that borderline tumours should be measured in 2 or more modalities for the most accurate size. On the other hand, overestimation of size can be used as an advantage for the surgeon - when planning treatment he may assume that the actual size is smaller. Involvement of the perinephric fat is a key point in planning NSS: if adjacent fat tissue infiltration is confirmed, radical nephrectomy becomes necessary. Perinephric spread of the tumour is the most difficult aspect in staging renal cell carcinoma. A major drawback of ultrasound is the fact that it cannot distinguish perinephric spread. Also, multidetector CECT is not free from limitations, being unable to sufficiently visualize involvement of surrounding fatty tissue and other adjacent organs. That is the main reason for decreased CT accuracy in renal cell carcinoma staging. At this point we found that $50 \%$ of tumour invasions were falsely analyzed, which leads us to the conclusion that if there is any risk of fat invasion CT and ultrasound cannot give accurate information. Further studies must be undertaken to search for possible factors to describe in $\mathrm{CT}$ examination to distinguish fat invasion.

In conclusion, using ultrasound and computed tomography, asymptomatic lesions are detected at an earlier stage when they are amenable to local excision with preservation of the normal adjacent parenchyma. With early detection, better differentiation and the smaller size of these accidentally diagnosed renal cell carcinomas, NSS becomes a real alternative to radical nephrectomy.

Analysis of our material has led to the conclusion that lesions localized in the lower kidney pole (confirmed both in ultrasound and $\mathrm{CT}$ ) have a better prognosis and more patients with such a diagnosis could be qualified for nephron-sparing surgery. Tumour location in the central part of the kidney is less advantageous compared to the lower pole due to the proximity of the renal pelvis which excludes the possibility of sparing surgery in many cases. Therefore when planning NSS, determination of the relationship of the tumour to the calico-pyelo system is highly important. Although tumour extension into the collecting system is considered as a relative contraindication for NSS, the necessity of its reconstruction might be a reason for performing radical nephrectomy. In our material, collecting system infiltration was seen in 33 cases based on CT examination, with no later confirmation in ultrasound, which is consistent with other studies [26].

Development of CT and MRI imaging has become a keystone in the preoperative planning and staging of renal cell carcinoma. CT remains the gold standard due to its low cost, accessibility and - which is very important - high accuracy [27].

\section{References}

1. Ljungberg B, Campbell SC, Cho HJ, Jacqmin D, Lee JE, Weikert S, Kiemeney LA. The epidemiology of renal cell carcinoma. Eur Urol 2011; 60: 615-21.

2. Lipworth L, Tarone RE, McLaughlin JK. The epidemiology of renal cell carcinoma. J Urol 2006; 176 (6 Pt 1): 2353-8.

3. Robson CJ, Churchill BM, Anderson W. The results of radical nephrectomy for renal cell carcinoma. J Urol 1969; 101: 297-301.

4. Leibovich BC, Blute M, Cheville JC, Lohse CM, Weaver AL, Zincke H. Nephron sparing surgery for appropriately selected renal cell carcinoma between 4 and $7 \mathrm{~cm}$ results in outcome similar to radical nephrectomy. J Urol 2004; 171: 1066-70.

5. Van Poppel H. Efficacy and safety of nephron-sparing surgery. Int J Urol 2010; 17: 314-326.

6. Hatcher PA, Anderson EE, Paulson DF, Carson CC, Robertson JE. Surgical management and prognosis of renal cell carcinoma invading the vena cava. J Urol 1991; 145: 20-23.

7. Hallscheidt PJ, Fink C, Haferkamp A, Bock M, Luburic A, Zuna I, Noeldge G, Kauffmann G. Preoperative staging of renal cell carcinoma with inferior vena cava thrombus using multidetector CT and MRI: prospective study with histopathological correlation., Journal Of Computer Assisted Tomography, vol. 29, no. 1, pp. 64-68, 2005.

8. Leveridge MJ, Bostrom PJ, Koulouris G, Finelli A, Lawrentschuk N. Imaging renal cell carcinoma with ultrasonography, CT and MRI. Nat Rev Urol 2010; 7: 311-25.

9. Russo P. Renal cell carcinoma: presentation, staging, and surgical treatment. Semin Oncol 2000; 27: 160-76.

10. Zagoria RJ, Dyer RB, Wolfman NT, Hinn GC, Chen YM. Radiology in the diagnosis and staging of renal cell carcinoma. Crit Rev Diagn Imaging 1990; 31: 81-115.

11. Konnak JW, Grossman HB. Renal cell carcinoma as an incidental find ing. J Urol 1985; 134: 1094-6.

12. Roos FC, Brenner W, Müller M, Schubert C, Jäger WJ, Thüroff JW, Hampel C. Oncologic long-term outcome of elective nephron-sparing surgery versus radical nephrectomy in patients with renal cell carcinoma stage pT1b or greater in a matched-pair cohort. Urology 2011; 77: 803-8.

13. Waalkes S, Becker F, Schrader AJ, Janssen M, Wegener G, Merseburger AS, Schrader M, Hofmann R, Stöckle M, Kuczyk MA. Is there a need to further subclassify PT2 renal cell cancers as implemented by the revised 7th TNM version? Eur Urol 2011; 59: 258-63.

14. Rodriguez R, Fishman EK, Marshall FF. Differential diagnosis and evaluation of the incidentally discovered renal mass. Semin Urol Oncol 1995; 13: 246-53.

15. Tsui KH, Shvarts O, Smith RB, Figlin R, de Kernion JB, Belldegrun A Renal cell carcinoma: prognostic significance of incidentally detected tumors. J Urol 2000; 163: 426-30.

16. Hallscheidt PJ, Bock M, Riedasch G, Zuna I, Schoenberg SO, Autschbach F, Soder M, Noeldge G. Diagnostic accuracy of staging renal cell carcinomas using multidetector-row computed tomography and magnetic resonance imaging: a prospective study with histopathologic correlation. J Comput Assist Tomogr 2004; 28: 333-9

17. McClennan BL, Deyoe LA. The imaging evaluation of renal cell carcinoma: diagnosis and staging. Radiol Clin North Am 1994; 32: 55-69.

18. Guo HF, Song Y, Na YQ. Value of abdominal ultrasound scan, CT and MRI for diagnosing inferior vena cava tumour thrombus in renal cell carcinoma. Chin Med J (Engl) 2009; 122: 2299-302. 
19. Jayson M, Sanders H. Increased incidence of serendipitously discovered renal cell carcinoma. Urology 1998; 51: 203-5.

20. Hélénon O, Correas JM, Balleyguier C, Ghouadni M, Cornud F. Ultrasound of renal tumors. Eur Radiol 2001; 11: 1890-901

21. Heidenreich A, Ravery V; European Society of Oncological Urology. Preoperative imaging in renal cell cancer. World J Urol 2004; 22: 307-15.

22. Zagoria RJ, Bechtold RE. The role of imaging in staging renal adenocarcinoma. Semin Ultrasound CT MR 1997; 18: 91-9.

23. Margreiter M, Marberger M. Current status of open partial nephrectomy. Curr Opin Urol 2010; 20: 361-4.

24. Zucchi A, Mearini E, Costantini E, Vivacqua C, Bini V, Mearini L. Clinical staging accuracy of renal tumors. Arch Ital Urol Androl 2004; 76 : 167-70.

25. Hellenthal NJ, Mansour AM, Hayn MH, Schwaab T. Renal cell carcinoma in octogenarians: nephron sparing surgery should remain the standard of care. J Urol 2011; 185: 415-20.

26. Graser A, Zech CJ, Stief CG, Reiser MF, Staehler M. Imaging renal cell carcinoma. Urologe A 2009; 48: 427-36.

27. Coll DM, Uzzo RG, Herts BR, Davros WJ, Wirth SL, Novick AC. 3-dimensional volume rendered computerized tomography for preoperative evaluation and intraoperative treatment of patients undergoing nephron sparing surgery. J Urol 1999; 161: 1097-102.

\section{Adres do korespondencji}

Sylwia Heinze-Paluchowska MD, PhD

Department of Diagnostic Imaging

Maria Skłodowska-Curie Institute of Oncology

Garncarska 11

31-115 Krakow, Poland

e-mail: sylwia.heinze-paluchowska@ifj.edu.pl

Submitted: 15.11 .2011

Accepted: $\quad 29.05 .2012$ 